\title{
Treatment of radiation-induced mucocutaneous toxicity
}

\author{
M. Becker-Schiebe ${ }^{1}$, F. Lordick², W. Hoffmann'1 \\ ${ }^{1}$ Klinikum Braunschweig, Department of Radiotherapy and Radiooncology, Braunschweig, Germany \\ ${ }^{2}$ Klinikum Braunschweig, 3rd Medical Department (Hematology and Medical Oncology), Braunschweig, Germany
}

Received 31 October 2011; accepted 28 November 2011

During radiotherapy $80 \%$ to $90 \%$ of all patients will develop some degree of inflammation symptoms, such as erythema, dry or wet desquamation, skin folds, or mucositis depending on radiation-and patient-related factors and the extent of irradiated skin or mucosal areas. Up to now radiation induced local reactions represent still an important toxicity factor. $\mathrm{Cu}-$ taneous and mucosal side effects may reduce the patient's compliance and can be limiting factors to follow radiotherapy protocols. Therefore, there is a high need for effective prophylactic and therapeutic treatments. Basically, guidelines recommend the avoidance of mechanical, chemical and thermal irritants, especially the exposure to high temperatures. To delay onset of radiodermatitis various preventive topicals may be applied like aqueous cream formula with or without antioxidative agents. In general, the treatment of radiodermatitis primarily should maintain moisture and skin permeability and consists of hydrophilic creams, antioxidative and antiinflammatory topicals. Hydrocolloid dressings may reduce and improve wound healing in grade 2 and 3 reactions. Supportive therapy of radiation-induced mucositis includes the maintenance of oral care protocols and adequate nutrition during the course of treatment. A sufficient oral health status is one of the most important factors for prevention of severe oral complications. The MASCC guidelines recommend furthermore the use of non-medicated rinses with saline or sodium bicarbonate 4 to 6 times daily. Further approaches suggest the use of local anaesthetics and systemic analgesics for severe mucositis. Besides local preventive agents and supportive care protocols, modern radiation treatment techniques remain the most promising intervention in reducing the degree of skin reactions.

Keywords: Irradiation, mucositis, radiodermatitis.

\section{Introduction}

Inflammatory skin reactions and mucositis are common toxicities in cancer patients undergoing radiotherapy.

Especially radiation induced mucositis in head and neck cancer patients remains a frequent side effect because

Correspondence: Martina Becker-Schiebe, MD, Klinikum Braunschweig, Department of Radiotherapy and Radiooncology, Celler Str. 38, 38114 Braunschweig, Germany.

E-mail: m.schiebe@klinikum-braunschweig.de of the more aggressive irradiation schedules. Furthermore concurrent application of chemotherapy and/or epidermal growth factor receptor (EGFR) targeted therapy does increase not only the therapeutic efficacy but also the frequency of side-effects significantly.

Oral mucositis grade I-II may affect more than $95 \%$ of head and neck cancer patients. The oral complications are often accompanied by pain and dysphagia and may become a dose limiting toxicity resulting in an unfavourable outcome of therapy. Therefore an intensive prophylactic and therapeutic supportive care management is warranted.

During exposure to therapeutic irradiation the most frequent complications are erythema and edema. It is estimated that more than $90 \%$ of patients undergoing curative irradiation with total doses $>55$ Gy will develop a radiodermatitis and approximately $10 \%$ of these patients encounter a grade 3 skin lesion before the scheduled end of RT [10]. Especially cutaneous side effects may both reduce the patient's compliance and quality of life. Preventive treatments are usually based on clinical experience and differ considerably between departments. The goal of management is to identify the patient at risk and to adopt strategies to delay onset of skin reactions.

\section{Supportive care and treatment recommendations}

\section{Radiodermatits}

The reaction of the skin to ionising radiation is highly complex and depends on numerous radiation-related, treatmentrelated, and patient-related factors. Previous studies [13] have tried to identify factors associated with an increased risk of acute radiation-induced toxicity. These include large irradiation volume, concomitant systemic treatment and comorbidities such as diabetes or hypertension. Treatment areas commonly sited as having a higher incidence of side-effects are those of head and neck, breast and chest wall fields and regions containing skin folds [1].

The patho-physiology of radiation-induced skin morbidities is understood as a secondary inflammatory reaction, possibly from the release of free radicals producing doubleand single-strand DNA breaks [6]. Acute cutaneous side-effects are defined as occurring within the first 6 months of therapy. The signs of toxicity are classified according to the 
Radiotherapy Oncology Group criteria (RTOG: acute skin radiation morbidity scoring criteria) [4]. Grade 1 toxicity is not usually experienced until total radiation doses of 20 to $25 \mathrm{~Gy}$.

During radiotherapy $80 \%$ to $90 \%$ of patients will develop some degree of inflammation symptoms, such as erythema, dry desquamation, moist desquamation pitting or oedema. In severe cases painful ulceration or necrosis may occur $[1,4]$.

Clinically, acute radiodermatitis presents as localised or confluent erythema, which seems to be correlated mostly with changes in arterioles. This obstruction leads to dermal infiltration and interstitial oedema. Therefore mechanically irritation of the dermis, sun exposure and skin traumas should be completely avoided. Several studies $[14,18]$ have shown that washing did not worse skin inflammation. Gentle washing should be permitted especially for patient's personal comfort.

Up to now the German guideline [21] does not recommend any prophylactic agents to prevent radiodermatitis. Different preventive recommendations are discussed controversially in the literature $[3,26]$. There are various concepts depending on personal preferences and institutional policy mainly based on retrospective data. Comparative studies did not demonstrate any improved prophylactic effect using hydrophilic emulsions with additional topicals such as trolamine, sucralfate, Theta Crème or aloe vera $[6,16,21]$. The majority of studies compared agents just in a retrospective setting. Recent trials suggest that antioxidative and anti-inflammatory products such as calendula and corticosteroids may have prophylactic impact in skin care [22]. The largest randomised evaluation of over 300 breast cancer patients demonstrated a significant reduction in grade $2 / 3$ dermatitis, comparing the effectiveness of calendula versus trolamine. In this randomised trial $30 \%$ of patients found calendula difficult to apply due to its more solid consistence and was not recommended at the end [15]. Two clinical studies [3,22] reported that low dose corticosteroids may be beneficial in reduction of itching and irritation. Inflammatory process may be alleviated, but skin thinning and skin atrophy may pose a problem using steroid topicals over a period of 8 weeks.

Actually, reports demonstrated first promising results using antioxidative agents such as silymarin or MAS065D (XClair ${ }^{\circ}$ ) in breast cancer patients. The beneficial effects of silymarin-based cream in reducing the rate of objective and subjective skin toxicity have been confirmed in one prospective trial evaluating 101 breast cancer patients [1]. Besides local agents modern radiation treatment techniques remain the most promising intervention in reducing the degree of skin reaction.

Therapeutic management of acute skin toxicity is based on clinical experience due to the inconsistencies in the clinical management and the absence of randomised trials [21]. Erythema grade 1 should be treated by hydrophilic cream to increase the skin's moisture and maintain skin permeability. [3]. Low dose corticosteroids ( $1 \%$ ) may reduce the severity of toxicity in case of itch, pain and burning. Up to now there is insufficient evidence to recommend specific dressings. Nevertheless silicon foam dressings have been shown to be effective compared to standard aqueous cream in breast cancer patients suffering from erythema in one observational trial [5]. Mean size of moist desquamation and ulceration was
Table 1: Summary of clinical suggestions for care of patients with radiodermatitis [3]

\section{Prevention}

- Avoidance of mechanical, thermal and chemical irritation, e.g. sun exposure

- Hydrophilic creams to improve moisture

- Three dimensional radiation treatment/IMRT/modern immobilisation systems

- Topicals (trolamine, sucralfate, Theta Crème, aloe vera): insufficient evidence

- Anti-inflammatory agents: calendula, local steroids: insufficient evidence

- Antioxidative agents - silymarin: preventive effect proven in one prospective trial

Treatment options (according to toxicity grade)

- Grade 1-3: hydrophilic creams

- Grade 2/3: corticosteroids (1\%)

- Grade 2-4: specific dressings: silicon foam, hydrocolloid

- Grade 4: hyperbaric oxygenation, skin grafting

- Local antiseptics in case of superinfection

- Antibiotic or antifungal therapy in case of superinfection

significantly smaller in patients receiving hydrocolloid dressings. Furthermore pain control and patient comfort did increase significantly in patients treated with hydrocolloid dressings for acute skin inflammation grades 2 and 3 [11]. In case of superinfection local antiseptics are indicated to eliminate the toxin, in severe cases systemic antibiotics should be used [26]. Chronic skin changes, such as necrosis and ulceration should be treated similar to those principles for burns and sometimes may require skin grafting. There are case reports suggesting that hyperbaric oxygenation may be safe and effective in managing radiation-induced late side-effects, such as soft tissue necrosis [20]. The summary of clinical suggestions for care of patients with radiodermatitis is shown in Table 1.

\section{Radiation induced mucosal injury of the oral cavity and head and neck region}

Radio \pm chemotherapy of the head and neck region may lead to inflammatory skin and mucosal reactions. The rapidly proliferating basal cells of the mucosal tissue are extremely radiosensitive. Two to three weeks after initiation of radiotherapy mucosal cell death of the basal tissue cause superficial ulcerations. The generation of free radicals by radiation results in DNA strand breaks. Furthermore in these cells activation of transcription factors such as NF kappa B leads to the up-regulation of genes that modulate apoptosis processes. Direct and indirect epithelial stem cell deaths result in a loss of repopulation capacity and support mucosal surface damage $[23,24]$.

The incidence of mucositis is influenced by numerous radiation-related factors and has been shown to arise with the total treatment dose, especially in patients receiving more than 50 Gy. Modern irradiation schedules using dose-escalation and acceleration are associated with higher toxicity rates in $29-45 \%$ of cases [24]. Oral mucositis occurs more often in patients receiving therapy for oropharyngeal or nasopharyngeal cancer concomitant to chemotherapy [8]. Pretreatment of cancer with induction chemotherapy may also alter the tol- 
erability of irradiation rendering skin and mucosa more susceptible to side-effects [7].

Mucositis grade I/II has now become one of to most important non-hematological toxicity affecting more than $90 \%$ of patients. Mucosal erythema is frequently followed by edema and ulceration, ranging from aphthous-like lesions to generalised desquamation. Inflammation may occur limited in range or can involve up to more than $50 \%$ of the oral cavity, most commonly in patients receiving combined therapy [8]. Mucosal ulceration may induce pain and dysphagia. Radiation-induced affection of the salivary glands contributes to xerostomia and reduces an important barrier against oral infections. This may lead to the reduced intake of oral nutrition resulting in the need for parenteral nutrition, feeding tube dependence, dehydration, weight loss and potentially severe infections and aspiration [12].

Prevention of oral mucositis includes the maintenance of oral care protocols and adequate nutrition during the course of treatment. Prophylactic percutaneous gastrostomy tube has been shown to be beneficial in patients receiving curative high dose radio-chemotherapy $[8,9]$.

A sufficient oral health status is important to reduce the incidence of severe oral complications. Prior to cancer therapy oral and teeth disorders such as dental plaque formation, periodontal diseases or other pathological sources of gingival irritation should be treated. Patients should avoid irritants such as alcohol and tobacco use. Oral hygiene measures such as soft tooth brushing and mouthwashes are recommended [17]. The RTOG and MASCC/ISOO [8] guidelines recommend furthermore the use of non-medicated rinses with saline or sodium bicarbonate 4 to 6 times daily. Non-randomised trials reported about 30 to $40 \%$ reduction in stomatitis for patients, who followed a daily preventive protocol with chlorhexidine $(0.2 \%)$ [19]. In a randomised clinical trial [23] preventive antimicrobial medication did not reduce the incidence of mucositis and is not recommended in the prophylactic setting.

Mouth rinses with anti-infective, antiphlogistic and anesthetic properties have been evaluated in several clinical studies, if mucositis develops [12]. In cases of superinfection antimicrobial topical agents such as amphotericin B or clotrimazole are usually curative. Because of the insufficient evidence of benefit, the guidelines of the MASCC do not recommend the use of radioprotective agents such as amifostine in head and neck cancer patients. Amifostine, as normal tissue protector during cytotoxic therapy, has been proven to reduce proctitis for rectal cancer patients only [2]. Topical benzydamine may be effective in pain control. It inhibits proinflammatory cytokines including TNF alpha and is characterised by non-steroidal anti-inflammatory, anesthetic and antimicrobial properties. Based on a multicenter randomised trial in head and neck cancer patients benzydamine is recommended in patients receiving moderate-dose adjuvant radiotherapy [9]. A favoured local mouth wash consists of antacid, diphenhydramine, to which lidocaine is added. Local formula with analgesic often has to be combined with systemic analgesics, including opiods for sufficient pain relief. Medication with antiulcerative properties such as prostaglandine or pentoxifylline were investigated with a marginal effect on mucositis. Growth factors, which not only stimulate precursor cells in the bone marrow but also affect the proliferation of other epithelial cells, have been applied for the management
Table 2: Summary of evidence-based clinical practice guidelines for care of patients with oral mucositis during radiotherapy (according to MASCC, [8])

\section{Prevention}

- Oral care protocols:

Prior to cancer therapy treatment of oral and teeth disorders

$>$ Brushing with soft tooth brush

Maintenance of oral hygiene

Non-medicated rinses (saline of sodium bicarbonate 4-6 times/ daily)

> Adequate nutrition (percutaneous gastrostomy tube in selected cases)

- Three dimensional radiation treatment/IMRT/modern technology

- Antimicrobial lozenges: evidence not proven

\section{Treatment}

- Patient-controlled analgesia (local and systemic)

- Benzydamine in patients receiving moderate-dose radiation therapy

- Antibiotic or antifungal therapy in case of superinfection

- Amifostine in head and neck cancer patients: evidence not proven

- Sucralfate: evidence not proven

- Growth factors (palifermin): evidence not proven

of oral mucositis. Palifermin (recombinant keratinocyte growth factor-1) reduced the incidence of grade 3 and 4 toxicities in patients with hematologic malignancies. Up to now this agent is currently under investigation in prospective trials including head and neck cancer patients [25].

The summary of evidence-based clinical practice guidelines for care of patients with oral mucositis during radiotherapy is shown in Table 2.

\section{Role of irradiation technique}

Up to now acute radiodermatitis and mucositis represent still an important toxicity factor for cancer patients, as it may cause important deterioration of the quality of life and patient's compliance with potential negative impact on RT treatment efficacy [21]. Using new technology and hyperfractionation schedules, the therapeutic ratio has been improved while the potential morbidity has decreased.

Modern radiation techniques, especially photon beams of higher energy, reduce skin dose, which provides better normal tissue protection [14]. Clinically the emphasis has been made on developing immobilisation procedures for protecting organs at risk. Mucosal side effects can be reduced significantly filling bolus materials between metallic dental restorations to minimise the dose from scattered radiation.

The introduction of individual immobilisation by means of mask-fixation may result in a relevant decrease in the uncertainty due to breathing motion and daily positioning errors. Especially diagonal thermoplastic materials are recommended for breast cancer patients to avoid bolus effects. To improve dose homogeneity and to avoid high doses in contiguous skin surface styrofoam wedge is indicated for pendulous breasts.

Modern radiation techniques such as 3D conformal therapy and IMRT (intensity modulated radiotherapy) allow to avoid 'hotspots' [14] and to reduce normal tissue reactions 
in a substantial number of patients. The new 'Rapid Arc' technology' is characterised by volumetric arcs that deliver a precisely sculpted 3D dose distribution with a single 360-degree rotation of the linear accelerator gantry and optimises dose conformity while significantly shortening treatment times. Recently, the value of high energy particles (protons) instead of X-rays (photons) is under investigation to minimise treatment sequela.

\section{Conclusion}

Although marked progress has been achieved in the management of side effects, further improvement is needed to optimise the supportive care management for cancer patients. Furthermore, research might focus on new strategies to prevent overall toxicity, by selecting the systemic treatment on tumour cells and protecting organs at risk in radiotherapy planning procedures. In future, further technical innovations are essential for the prevention of radiation toxicity.

\section{Conflict of interest}

The authors declare that there is no conflict of interest.

\section{References}

[1] Becker-Schiebe M, Mengs U, Schaefer M, Bulitta M, Hoffmann W. Topical use of a silymarin-based preparation to prevent radiodermatitis: results of a prospective study in breast cancer patients. Strahlenther Onkol, 187: 485-91, 2011.

[2] Ben-Josef E, Han S, Tobi M, et al. Intrarectal application of amifostine for the prevention of radiation induced rectal injury. Semin Radiat Oncol, 12: 81-5, 2002.

[3] Bolderston A, Lloyd N, Wong R, Holden L, Robb-Blenderman L. The prevention and management of acute skin reactions related to radiation therapy: a systematic review and practice guideline. Supportive Care Cancer, 14: 802-17, 2006.

[4] Cox JD, Stetz J, Pajak TF. Toxicity criteria of the Radiation Therapy Oncology Group (RTOG) and the European Organization for Research and Treatment of Cancer (EORTC). Int J Radiat Oncol Biol Phys, 31: 1341-6, 1995.

[5] Diggelmann K, Zytkovicz AE, Tuaiine JM, Bennett NC, et al. Mepilex Lite dressing for the management of radiation induced erythema: a systematic inpatient controlled clinical trial. Brit J Radiol, 83: 971-8, 2010.

[6] Falkowski, Trouillas P, Duroux JL, Bonnetblanc JM, Clavère P. Radiodermatitis prevention with sucralfate in breast cancer: fundamental and clinical studies. Support Care Cancer, 19: 57-65, 2011.

[7] Hoffmann W, Rodemann HP, Schmidberger H, Weiss E, Bamberg M. Enhanced muco-cutaneous toxicity of radiotherapy in a patient with chronic GVHD and secondary malignancy. Annal Oncol, 5: 377-9, 1994.

[8] Keefe DM, Schubert MM, Elting SE, et al. Updated clinical practice guidelines for the prevention and treatment of mucositis. Cancer, 109: 820-31, 2007.
[9] Lalla RV, Schubert MM, Bensadoun RJ, Keefe D. Anti-inflammatory agents in the management of alimentary mucositis. Support Care Cancer, 14: 558-65, 2006.

[10] López E, Núñez MI, Guerrero MR, et al. Breast cancer acute radiotherapy morbidity evaluated by different scoring systems. Breast Cancer Res Treat, 73: 127-34, 2002.

[11] Mak SS, Lolassiotis A, Wan W, et al. The effects of hydrocolloid dressing and gentian violet on radiation induced moist desquamation wound healing. Canc Nurs, 23: 220-9, 2000.

[12] McGuire DB, Correa ME, Johnson J, Wienandts P. The role of basic oral care and good clinical practice principles in the management of oral mucositis. Support Care Cancer, 14: 541-7, 2006.

[13] Moran MS, Haffty BG. Radiation techniques and toxicities for locally advanced breast cancer. Semin Radiat Oncol, 19(4): 244-55, 2009.

[14] Pignol J, Olivotto I, Rakovitch E, Gardner S, Sixel K, Beckham W, et al. A multicenter randomized trial of breast intensity-modulated radiation therapy to reduce acute radiation dermatitis. J Clin Oncol 26: 2085-92, 2008.

[15] Pommier P, Gomez F, Sunyach MP, D’Hombres A, Carrie C, Montbarbon X. Phase III randomized trial of Calendula officinalis compared with trolamine for the prevention of acute dermatitis during irradiation for breast cancer. J Clin Oncol, 22(8): 1447-53, 2004.

[16] Röper B, Kaisig D, Auer F, Mergen E, Molls M. Thêta-Cream versus Bepanthol lotion in breast cancer patients under radiotherapy. A new prophylactic agent in skin care? Strahlenther Onkol, 180(5): 315-22, 2004.

[17] Rosenthal DI, Trotti A. Strategies for managing radiation induced mucositis in head and neck cancer patients. Semin Radiat Oncol, 19: 29-34, 2009.

[18] Roy I, Fortin A, Larochelle M. The impact of skin washing with water and soap during breast irradiation. A randomized study. Radiother Oncol, 58: 333-9, 2001.

[19] Rubenstein E, Peterson D, Schubert M, et al. Clinical practice guidelines for the prevention and treatment of cancer therapy-induced oral and gastrointestinal mucositis. Cancer, 100(Suppl.): 2026-46, 2004.

[20] Safra t, Gutman G, Fishlev G, et al. Improved quality of life with hyperbaric oxygen therapy in patients with persistent pelvic radiationinduced toxicity. Clin Oncol, 20: 284-7, 2008.

[21] Sauer R (Koordinator Leitlinie Mammakarzinom), Budach W, Dunst J, Feyer P, Haase W, Harms W, Sautter-Bihl M, Souchon R, Wenz F. Leitlinien in der Radioonkologie. Leitlinie Radiotherapie des Mammakarzinoms. Strahlenther Onkol, 182(1): 17-8, 2006.

[22] Shukla PN, Gairola M, Mohanti BK, Rath GK. Prophylactic beclomethasone spray to the skin during postoperative radiotherapy of carcinoma breast: A prospective randomized study. Indian J Cancer, 43(4): 180-4, 2006.

[23] Stokman MA, Spijkervet FK, Burlage FR, et al. Oral mucositis and selective elimination of oral flora in head and neck cancer patients receiving radiotherapy: A double-blind randomised clinical trial. $\mathrm{Br}$ J Cancer, 88: 1012-6, 2003.

[24] Vera-Llonch M, Oster G, Hagiwara M, et al. Oral mucositis in patients undergoing radiation treatment for head and neck carcinoma. Cancer, 106: 329-36, 2006.

[25] Von Bultzingslowen I, Brennan MT, Spijkervet FK, et al. Growth factors and cytokines in the prevention and treatment of oral and gastrointestinal mucositis Support Care Cancer, 14: 519-27, 2006.

[26] Zimmermann J, Budach W, Dörr W. Individual skin care during radiotherapy. Strahlenther Onkol, 174: 74-7, 1998. 